\title{
Erbium triflate: a valuable and non-toxic catalyst for the synthesis of acylals and enol esters
}

\author{
Renato Dalpozzo, ${ }^{\mathrm{a}}$ Antonio De Nino, ${ }^{\mathrm{a} *}$ Loredana Maiuolo, ${ }^{\mathrm{a}}$ Monica Nardi, ${ }^{\mathrm{a}}$ Antonio \\ Procopio, ${ }^{\text {b Beatrice Russo, }}{ }^{\mathrm{b}}$ and Antonio Tagarelli ${ }^{\mathrm{a}}$ \\ ${ }^{a}$ Dipartimento di Chimica, Università della Calabria, Ponte Bucci, cubo 12C, 87036 \\ Arcavacata di Rende (Cs), Italy \\ ${ }^{b}$ Dipartimento di Scienze Farmaco-Biologiche, Università della Magna Graecia, Complesso \\ Ninì Barbieri, 88021 Roccelletta di Borgia (Cz), Italy \\ E-mail: denino@unical.it
}

Dedicated to Professor Giuseppe Bartoli on his 65th birthday

\begin{abstract}
$\operatorname{Er}(\mathrm{OTf})_{3}$ was found to be an effective Lewis acid catalyst for the conversion of aldehydes into acylals. Unlike most of the other known catalysts, it is able to give acylals from acetic anhydride as well as other anhydrides. Moreover, it can react with cyclic ketones to give unstable acylals, which can be isolated as enol esters. Other advantages are the very low loading of catalyst, excellent chemoselectivity, and solvent-free and mild conditions.
\end{abstract}

Keywords: Erbium(III) triflate, Lewis acid catalyst, acylals, aldehydes, ketones

\section{Introduction}

Acylals (geminal diacetates) or gem-bis(acyloxy)-alkanes have been considered to be important protecting groups in organic synthesis, as alternatives to acetals ${ }^{1}$ for protection of aldehydes, owing to their stability towards aqueous acids ${ }^{2}$ and bases. ${ }^{3}$ They are also used as substrates for nucleophilic substitution reactions. ${ }^{4}$ Their utility as cross-linking reagents for cellulose in cotton, ${ }^{5}$ as well as activators in the composition of bleaching mixture used for the treatment of winestrained fabrics is also well established. ${ }^{6}$ Various catalysts have reported for their synthesis. ${ }^{7,8}$ Many of these reagents are highly corrosive and difficult to handle, while some Lewis acid catalysts are rather expensive and moisture sensitive. Some procedures require the use of a large excess of acetic anhydride to effect acylal formation. Further, there are very few reports in the literature on the formation of acylals using other anhydrides, ${ }^{7 \mathrm{k}, \mathrm{u}}$ and finally, despite the plethora of synthetic methods for formation of aldehyde acylals, no data are reported on the reaction of 
ketones. Given the synthetic utility of acylals, newer reagents that are inexpensive, non-toxic, chemoselective, and effective for acylal formation with a variety of anhydrides would provide a valuable addition to the literature.

In the last few years, we have been interested in many reactions catalyzed by Lewis acids. ${ }^{9}$ However, the lanthanoid family possesses the interesting feature of a regular variation of their properties along the series, which can be tuned through a proper choice of the cation. Therefore, many examples exist where rare-earth metal triflates are used as Lewis acid catalysts with efficacy varying from one reaction to another. ${ }^{10}$ Recently, the relative Lewis acidities of lanthanoid(III) triflates were evaluated by the use of tandem mass spectrometry, ${ }^{11}$ and in that study erbium(III) proved to be one of the most active cations. In fact its $\mathrm{pK}_{\mathrm{H}}$ and WERC values are 7.9 and $1.4 \times 10^{8}$ respectively, which are perfectly in accordance to Kobayashi statement. ${ }^{12}$ Nevertheless, Er(III) triflate has so far been neglected among lanthanide triflates, as useful and environmentally friendly catalysts for many acid-catalyzed reactions, such as the acylation of alcohols, ${ }^{13}$ or dioxolane protection removal, ${ }^{14}$ epoxide rearrangement, ${ }^{15}$ and the synthesis of $\mathrm{C}$ pseudoglycals. $^{16}$

In this paper we demonstrate that erbium triflate is a very valuable catalyst for the synthesis of both acylals of aldehydes and enol esters of ketones.

\section{Results and Discussion}

The most commonly used reagent for acylal formation is acetic anhydride, which results in the formation of 1,1-diacetates. To set up reaction conditions, three sample runs were tested: (i) benzaldehyde (1a) and acetic anhydride (2a) in acetonitrile without catalyst; (ii) $1 \mathbf{a}$ and $\mathbf{2 a}$ in acetonitrile and $0.1 \%$ erbium triflate; (iii) $1 \mathbf{a}$ and $\mathbf{2 a}$ and $0.1 \%$ erbium triflate in solvent free conditions (Scheme 1).

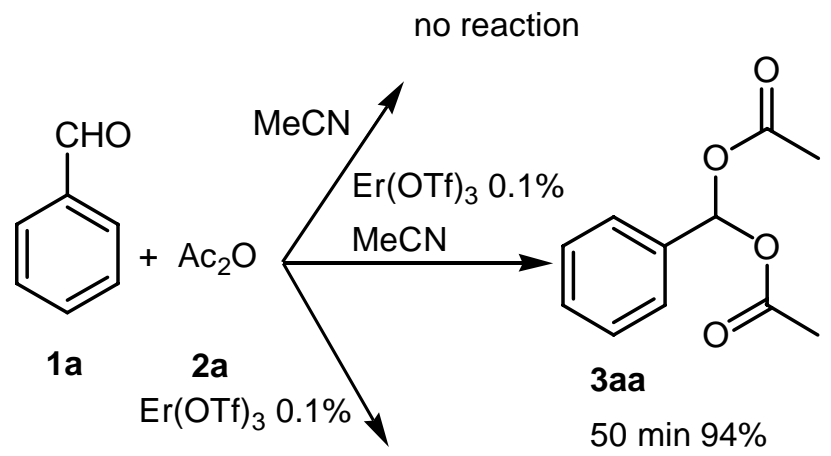

3aa

$15 \min 95 \%$

\section{Scheme 1}


As expected, the presence of a catalyst was shown to be essential for the occurrence of the reaction: in fact, in Run (i) the reagents were unaffected after $48 \mathrm{~h}$. On the other hand, in the presence of the catalyst, the reaction was almost complete, and when solvent-free conditions are used the reaction times are much shorter. To exclude catalysis of adventitious of $\mathrm{TfOH}$ in $\operatorname{Er}(\mathrm{OTf})_{3}$, we performed a sample reaction in the presence of $0.1 \%$ of DIPEA without loss of reactivity.

Since these conditions are also more environmentally benign, acetic acid from the excess of anhydride being the only by-product, these conditions were chosen to extend the reaction to a series of aldehydes (1b-m) (Table 1).

Table 1. Reaction of aldehydes with acetic anhydride (2a) in solvent-free conditions and with $0.1 \%$ erbium(III) triflate, at RT

\begin{tabular}{|c|c|c|c|c|}
\hline Run & Aldehyde (1) & Diacylal & Time (min) & Yield $(\%)$ \\
\hline $\mathrm{a}$ & $\mathrm{PhCHO}$ & $\mathrm{PhCH}(\mathrm{OAc})_{2}($ (3aa $)$ & 15 & 95 \\
\hline $\mathrm{b}$ & $4-\mathrm{ClC}_{6} \mathrm{H}_{4} \mathrm{CHO}$ & 4- $\mathrm{ClC}_{6} \mathrm{H}_{4} \mathrm{CH}(\mathrm{OAc})_{2}$ (3ba) & 15 & 98 \\
\hline $\mathrm{c}$ & $2-\mathrm{ClC}_{6} \mathrm{H}_{4} \mathrm{CHO}$ & $2-\mathrm{ClC}_{6} \mathrm{H}_{4} \mathrm{CH}(\mathrm{OAc})_{2}$ (3ca) & 20 & 97 \\
\hline$d$ & $4-\mathrm{NO}_{2} \mathrm{C}_{6} \mathrm{H}_{4} \mathrm{CHO}$ & $4-\mathrm{NO}_{2} \mathrm{C}_{6} \mathrm{H}_{4} \mathrm{CH}(\mathrm{OAc})_{2}$ (3da) & 15 & 99 \\
\hline e & $2-\mathrm{NO}_{2} \mathrm{C}_{6} \mathrm{H}_{4} \mathrm{CHO}$ & $2-\mathrm{NO}_{2} \mathrm{C}_{6} \mathrm{H}_{4} \mathrm{CH}(\mathrm{OAc})_{2}$ (3ea) & 20 & 97 \\
\hline $\mathrm{f}$ & $4-\mathrm{MeC}_{6} \mathrm{H}_{4} \mathrm{CHO}$ & $4-\mathrm{MeC}_{6} \mathrm{H}_{4} \mathrm{CH}(\mathrm{OAc})_{2}$ (3fa) & 15 & 94 \\
\hline g & $4-\mathrm{MeOC}_{6} \mathrm{H}_{4} \mathrm{CHO}$ & 4- $\mathrm{MeOC}_{6} \mathrm{H}_{4} \mathrm{CH}(\mathrm{OAc})_{2}$ (3ga) & 15 & 96 \\
\hline $\mathrm{h}$ & $4-\mathrm{OHC}_{6} \mathrm{H}_{4} \mathrm{CHO}$ & $4-\mathrm{AcOC}_{6} \mathrm{H}_{4} \mathrm{CH}(\mathrm{OAc})_{2}$ (3ha) & 10 & 98 \\
\hline $\mathrm{i}$ & $2-\mathrm{OHC}_{6} \mathrm{H}_{4} \mathrm{CHO}$ & $2-\mathrm{AcOC}_{6} \mathrm{H}_{4} \mathrm{CH}(\mathrm{OAc})_{2}$ (3ia) & 10 & 91 \\
\hline $\mathrm{j}$ & $4-\mathrm{Me}_{2} \mathrm{NC}_{6} \mathrm{H}_{4} \mathrm{CHO}$ & $4-\mathrm{Me}_{2} \mathrm{NC}_{6} \mathrm{H}_{4} \mathrm{CH}(\mathrm{OAc})_{2}$ (3ja) & 2160 & 0 \\
\hline $\mathrm{k}$ & $\mathrm{PhCH}_{2} \mathrm{CHO}$ & $\mathrm{PhCH}_{2} \mathrm{CH}(\mathrm{OAc})_{2}$ (3ka) & 5 & 98 \\
\hline 1 & $\mathrm{PhCH}=\mathrm{CHCHO}$ & $\mathrm{PhCH}=\mathrm{CHCH}(\mathrm{OAc})_{2}$ (3la) & 10 & 94 \\
\hline $\mathrm{m}$ & $\mathrm{C}_{5} \mathrm{H}_{11} \mathrm{CHO}$ & $\mathrm{C}_{5} \mathrm{H}_{11} \mathrm{CH}(\mathrm{OAc})_{2}$ (3ma) & 5 & 98 \\
\hline
\end{tabular}

Reaction was complete in few minutes and smoothly afforded the corresponding acylals at RT in excellent yields. Side-product formation was not observed in any reaction. Hydroxy groups were also acetylated to afford the corresponding triacetates ( $\mathbf{3} \mathbf{~ h a , ~ i a ) . ~}$

The presence of electron-donating or electron-withdrawing groups on the aromatic ring of benzaldehyde, irrespective of their position, did not substantially modify yields or reaction times. On the other hand, $\mathrm{AlPW}_{12} \mathrm{O}_{40}$ works better with aldehydes carrying electron-donating groups, ${ }^{7 \mathrm{r}}$ whereas $\mathrm{Bi}\left(\mathrm{NO}_{3}\right)_{3} .5 \mathrm{H}_{2} \mathrm{O}$ is better with electron-withdrawing ones. ${ }^{7 \mathrm{u}}$

As observed with other catalysts $\left.\left(\mathrm{InBr}_{3}\right),{ }^{7 \mathrm{w}} \mathrm{In}(\mathrm{OTf})_{3},{ }^{7 \mathrm{y}} \mathrm{AlPW}_{12} \mathrm{O}_{40},{ }^{7 \mathrm{r}} \mathrm{H}_{6} \mathrm{P}_{2} \mathrm{~W}_{18} \mathrm{O}_{62} .24 \mathrm{H}_{2} \mathrm{O}\right),{ }^{7 \mathrm{q}}$ 4-(N,N-dimethylamino)benzaldehyde, (1j), was deactivated and remained unaffected after 36 h. ${ }^{17}$

In order to show the merit of the present work, the results for 4-nitro- (1d) and 4-hydroxybenzaldehyde (1h) can be compared with the catalyst amount, the reaction time and the yields recently reported in other protocols (Table 2). Er(OTf) ${ }_{3}$ promotes both reactions more effectively 
than all the other catalysts, as far as the amount of catalyst and reaction times are concerned. Only $\mathrm{InBr}_{3}$ leads to 3da in comparable yields and efficiency, but it is less effective in the synthesis of 3ha. ${ }^{7 \mathrm{w}}$

The utility of the present method was further extended by employing it in the formation of other gem-diacyls. The reaction proceeded without loss of efficiency. Only benzoic anhydride (2e) lowered the yields (Table 3), very likely owing to electronic effects of phenyl rings on the anhydride moiety.

Table 2. Comparison of the effect of catalyst for gem-diacetate synthesis

\begin{tabular}{|c|c|c|c|c|}
\hline \multicolumn{5}{|c|}{ 4-Nitrobenzaldeyde } \\
\hline Catalyst & mol. \% & Time & Yield (\%) & Ref \\
\hline $\operatorname{Er}(\mathrm{OTf})_{3}$ & 0.1 & $15 \mathrm{~min}$ & 99 & - \\
\hline $\mathrm{I}_{2}$ & 10 & $2 \mathrm{~h}$ & 99 & $7 \mathrm{e}$ \\
\hline $\mathrm{InCl}_{3}$ & 10 & $4 \mathrm{~h}$ & 88 & $7 \mathrm{~m}$ \\
\hline $\mathrm{InBr}_{3}$ & 0.1 & $15 \mathrm{~min}$ & 99 & $7 \mathrm{w}$ \\
\hline $\operatorname{In}(\mathrm{OTf})_{3}$ & 0.1 & 15 & 99 & $7 y$ \\
\hline LiOTf & 20 & $15 \mathrm{~h}$ & 94 & $7 \mathrm{t}$ \\
\hline $\mathrm{Cu}(\mathrm{OTf})_{2}$ & 2.5 & $4 \mathrm{~h}$ & 94 & $7 \mathrm{~h}$ \\
\hline $\mathrm{Sc}(\mathrm{OTf})_{3}$ & 2 & $10 \mathrm{~min}$ & 99 & $7 f$ \\
\hline $\mathrm{ZrCl}_{4}$ & 5 & $30 \mathrm{~min}$ & 92 & $7 \mathrm{~s}$ \\
\hline $\mathrm{AlPW}_{12} \mathrm{O}_{40}$ & 0.1 & $45 \mathrm{~min}$ & 89 & $7 \mathrm{r}$ \\
\hline $\mathrm{LiBF}_{4}$ & 100 & $7.5 \mathrm{~h}$ & 80 & $7 \mathrm{k}$ \\
\hline $\mathrm{Cu}\left(\mathrm{BF}_{4}\right)_{2} \cdot x \mathrm{H}_{2} \mathrm{O}$ & 1 & $3 \mathrm{~min}$ & 92 & $7 v$ \\
\hline CAN & 10 & $24 \mathrm{~h}$ & 96 & $7 p$ \\
\hline $\mathrm{Bi}(\mathrm{OTf})_{3} \cdot x \mathrm{H}_{2} \mathrm{O}$ & 0.1 & $1.5 \mathrm{~h}$ & $95^{a}$ & $7 \mathrm{j}$ \\
\hline $\mathrm{Bi}\left(\mathrm{NO}_{3}\right)_{3} .5 \mathrm{H}_{2} \mathrm{O}$ & 10 & $2.5 \mathrm{~h}$ & $85^{a, b}$ & $7 \mathrm{u}$ \\
\hline $\mathrm{H}_{6} \mathrm{P}_{2} \mathrm{~W}_{18} \mathrm{O}_{62} .24 \mathrm{H}_{2} \mathrm{O}$ & 1 & $30 \mathrm{~min}$ & 92 & $7 q$ \\
\hline $\mathrm{Zr}\left(\mathrm{MePO}_{3}\right)_{1.2}\left(\mathrm{O}_{3} \mathrm{PC}_{6} \mathrm{H}_{4} \mathrm{SO}_{3} \mathrm{H}\right)_{0.8}$ & 0.05 & $24 \mathrm{~min}$ & 91 & 7o \\
\hline \multicolumn{5}{|c|}{ 4-Hydroxybenzaldehyde } \\
\hline $\operatorname{Er}(\mathrm{OTf})_{3}$ & 0.1 & $10 \mathrm{~min}$ & 98 & - \\
\hline $\operatorname{In}(\mathrm{OTf})_{3}$ & 0.1 & $40 \mathrm{~min}$ & $96^{c}$ & $7 y$ \\
\hline $\mathrm{InBr}_{3}$ & 0.1 & $12 \mathrm{~h}$ & 96 & $7 \mathrm{w}$ \\
\hline $\mathrm{Bi}(\mathrm{OTf})_{3} \cdot x \mathrm{H}_{2} \mathrm{O}$ & 0.1 & $20 \mathrm{~min}$ & 98 & $7 \mathrm{j}$ \\
\hline $\mathrm{Bi}\left(\mathrm{NO}_{3}\right)_{3} .5 \mathrm{H}_{2} \mathrm{O}$ & 10 & $-{ }^{d}$ & 91 & $7 \mathrm{u}$ \\
\hline $\mathrm{H}_{6} \mathrm{P}_{2} \mathrm{~W}_{18} \mathrm{O}_{62} \cdot 24 \mathrm{H}_{2} \mathrm{O}$ & 1 & $30 \mathrm{~min}$ & $95^{c}$ & $7 q$ \\
\hline
\end{tabular}

${ }^{a}$ Refers to 2-nitrobenzaldehyde. ${ }^{b}$ At reflux. ${ }^{c}$ Refers to 3-hydroxybenzaldehyde. ${ }^{d}$ Two-step procedure: (i) formation of 4-acetoxybenzaldehyde without catalyst, (ii) conversion to gemdiacetate. The one-step procedure gives rise to complex product mixture. 
Table 3. Reaction of 1a with anhydrides in solvent-free conditions and with $0.1 \%$ erbium(III) triflate, at RT.

\begin{tabular}{|c|c|c|c|c|}
\hline Run & Anhydride (2) & Diacylal (3) & Time $(\min )$ & Yield $(\%)$ \\
\hline $\mathrm{a}$ & $(\mathrm{MeCO})_{2} \mathrm{O}$ & $\mathrm{PhCH}(\mathrm{OCMe})_{2}$ (3ab) & 15 & 95 \\
\hline $\mathrm{b}$ & $(\mathrm{EtCO})_{2} \mathrm{O}$ & $\mathrm{PhCH}(\mathrm{OCEt})_{2}(\mathbf{3 a c})$ & 10 & 96 \\
\hline $\mathrm{c}$ & $(i-\operatorname{PrCO})_{2} \mathrm{O}$ & $\mathrm{PhCH}(\mathrm{OC}-i-\mathrm{Pr})_{2}$ (3ad) & 10 & 99 \\
\hline $\mathrm{d}$ & $\left(\mathrm{Me}_{3} \mathrm{CO}\right)_{2} \mathrm{O}$ & $\mathrm{PhCH}\left(\mathrm{OCMe}_{3}\right)_{2}(\mathbf{3 a e})$ & 10 & 99 \\
\hline $\mathrm{e}$ & $(\mathrm{PhCO})_{2} \mathrm{O}$ & $\mathrm{PhCH}(\mathrm{OCPh})_{2}$ (3af) & 150 & 45 \\
\hline
\end{tabular}

To our knowledge, gem-diacylals of benzaldehyde are only reported, under $\mathrm{Yb}(\mathrm{OTf})_{3}$ catalysis, as intermediates for the reductive esterification of aldehydes. ${ }^{18}$ However, a solvent $(\mathrm{MeCN}), 10 \% \mathrm{~mol}$ of catalyst per mole of aldehyde, and longer reaction times $(12 \mathrm{~h})$ are required. $\mathrm{ZrCl}_{4}$ is able to give gem-dipivalates in high yields at $\mathrm{RT}$, but with 5 mol.\% of catalyst per mole of aldehyde. ${ }^{7 \mathrm{~s}}$ Lithium tetrafluoroborate leads to gem-dipivalates at higher temperatures $\left(60{ }^{\circ} \mathrm{C}\right)$ and longer reaction times $(25 \mathrm{~h}){ }^{7 \mathrm{k}}$ The gem-butyrate and -isobutyrate of benzaldehyde and 4-chlorobenzaldehyde were also obtained under $\mathrm{Bi}\left(\mathrm{NO}_{3}\right)_{3} .5 \mathrm{H}_{2} \mathrm{O}$ catalysis, with longer reaction times $(4-16 \mathrm{~h})$, higher catalyst amounts $(5-10 \% \mathrm{~mol})$, and lower yields $(68-91 \%){ }^{7 \mathrm{u}}$

All the known catalysts were unable to give ketone gem-diacylals, apart from $\mathrm{Zr}\left(\mathrm{MePO}_{3}\right)_{1.2}\left(\mathrm{O}_{3} \mathrm{PC}_{6} \mathrm{H}_{4} \mathrm{SO}_{3} \mathrm{H}\right)_{0.8}$ which is reported to give cyclohexanone 1,1-diacetate in low yields. ${ }^{70}$ Therefore a high stereoselectivity towards aldehydes in competitive acylation reactions is limited by the applicability of the reaction.

A competition experiment was attempted for the acylation of $\mathbf{1 a}$ in the presence of cyclohexanone (1n) under solvent-free conditions, at RT in the presence of $0.1 \%$ mol. of erbium triflate. After 15 minutes, only 1a was acylated, while 1n was still unaffected. After 24 h, however, GC-MS analysis of the reaction mixture revealed, together with 1n and 3aa, the presence of two new peaks, whose fragmentation patterns were in agreement with cyclohexanone 1,1-diacetate (3na) and 1-acetoxycyclohexene (4na). Therefore, 2a and 1n were allowed to react, under the same reaction conditions. After $24 \mathrm{~h}$, GC-MS analysis of the mixture revealed the presence of $\mathbf{1 n}$, 3na and 4na in comparable amounts. Longer reaction times did not modify the relative percentages of the three products. Workup of the reaction, however, allowed the isolation only of starting $\mathbf{1 n} .^{19}$

The reaction mixture was then refluxed for 30 minutes and starting material was completely converted into the enol ester 4na in $75 \%$ isolated yield (Scheme 2). 


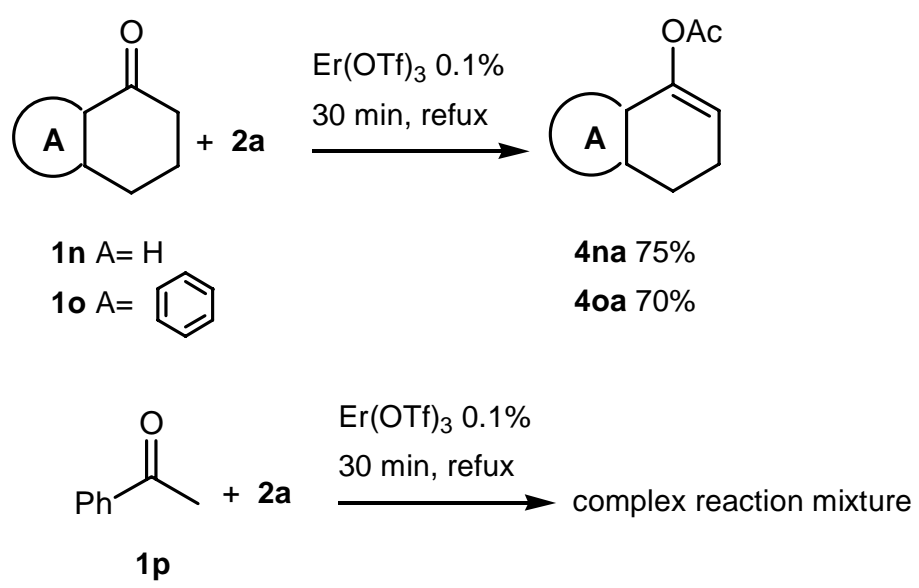

\section{Scheme 2}

Analogously $\alpha$-tetralone 10 afforded the expected enol acetate 4oa in comparable yields. Acetophenone 1p, instead, gave rise to a complex reaction mixture, where dimers and oligomers from aldol condensation, together, enol ether were recognized by GC-MS analysis.

1,1-Diacylals of ketones are, therefore, very unstable under employed acidic conditions and cannot be isolated. ${ }^{20}$ When formed they must be converted in more stable compounds such as enol ester by acetic acid elimination. However, aldol condensation side-reaction occurs with open chain ketones during elimination process.

\section{Conclusions}

$\operatorname{Er}(\mathrm{OTf})_{3}$ was found to be a novel Lewis acid catalyst for the conversion of aldehydes into acylals. Erbium was revealed as the most efficient among the known catalysts, since it is able to give acylals of anhydrides other than acetic. Unlike other catalysts, erbium triflate can react with cyclic ketones to give unstable ketone acylals, which can be isolated as enol esters. Other advantages are the very low loading of catalyst, excellent chemoselectivity, and solvent-free and mild conditions.

\section{Experimental Section}

General Procedures. ${ }^{1} \mathrm{H}$ - and ${ }^{13} \mathrm{C}-\mathrm{NMR}$ spectra were recorded with a Bruker WM300 instrument, at $300 \mathrm{MHz}$ and $75 \mathrm{MHz}$ respectively. Reactions were monitored by a GC-MS Hewlett-Packard workstation, formed by a GC-HP 6890 (30-m HPS capillary column, $1 \mathrm{~mL} / \mathrm{min}$ He as carrier gas) and an HP 5973 mass detector. Commercial products (Aldrich or Fluka) were used without further purification. Commercial $\mathrm{Er}(\mathrm{OTf})_{3} \cdot 6 \mathrm{H}_{2} \mathrm{O}$ was stored in an oven at $110^{\circ} \mathrm{C}$ or over $\mathrm{P}_{2} \mathrm{O}_{5}$ at RT. RT denotes room temperature. 


\section{Representative procedure for formation of acylals using acetic anhydride}

Aldehydes. A solution of benzaldehyde (1a) $(0.50 \mathrm{~g}, 4.72 \mathrm{mmol})$ in acetic anhydride (2a) $(1 \mathrm{~mL})$ was stirred and $\operatorname{Er}(\mathrm{OTf})_{3}(3 \mathrm{mg}, 0.0048 \mathrm{mmol}, 0.1 \mathrm{~mol} \%$ ) was added at RT. The reaction progress was monitored by TLC. After $15 \mathrm{~min}$, a solution of $10 \% \mathrm{NaHCO}_{3}$ was added and the mixture was extracted with ether $(2 \times 2 \mathrm{~mL})$. The combined organic layers were dried $\left(\mathrm{Na}_{2} \mathrm{SO}_{4}\right)$. The solvents were removed on a rotary evaporator to give $0.93 \mathrm{~g}(95 \%)$ of benzylidene diacetate (3аa).

Ketones. A solution of cyclohexanone (1n) $(0.50 \mathrm{~g}, 5.10 \mathrm{mmol})$ in 2a $(1 \mathrm{~mL})$ was stirred as $\mathrm{Er}(\mathrm{OTf})_{3}(3.1 \mathrm{mg}, 0.005 \mathrm{mmol}, 0.1 \mathrm{~mol} \%$ ) was added at RT. The mixture was then refluxed and the progress monitored by TLC. After $30 \mathrm{~min}$, a solution of $10 \% \mathrm{NaHCO}_{3}$ was added and the mixture extracted with ether $(2 \times 2 \mathrm{~mL})$. The combined organic layers were dried $\left(\mathrm{Na}_{2} \mathrm{SO}_{4}\right)$. The solvents were removed on a rotary evaporator to give $0.53 \mathrm{~g}(75 \%)$ of 1 -acetoxycyclohexene (4na).

All isolated compounds gave satisfactory microanalyses, EI-MS and NMR spectra comparable with reported ones: 3aa, 3ba, 3fa, 3la, 3ac; ${ }^{7 \mathrm{u}}$ 3ca, 3da, 3ea, 3ma; ${ }^{7 \mathrm{w}}$ 3ga, 3ha; $^{7 \mathrm{j}}$ 3ka; ${ }^{21}$ 3ia; ${ }^{7 \mathrm{q}}$ 3ab, 3ad, 3ae; ${ }^{18}$ 4na; ${ }^{22}$ 4oa. $^{23}$

\section{References and footnotes}

1. Greene, T. W.; Wuts, P. G. M. Protective Groups in Organic Synthesis, 3rd Edn., Wiley: New York, 1999; p 306.

2. Kochhar, K. S.; Bal, B. S.; Deshpande, R. P.; Rajadhyaksha, S. N.; Pinnick, H. W. J. Org. Chem. 1983, 48, 1765.

3. Gregory, M. J. J. Chem. Soc. B 1970, 1201.

4. Gallucci, R. R.; Going, R. C. J. Org. Chem. 1982, 47, 3517. (b) Ghribi, A.; Alexakis; A. Normant, J. F. Tetrahedron Lett. 1984, 25, 3079. (c) Trost, B. M.; Vercauteren, J. Tetrahedron Lett. 1985, 26, 131. (d) Narayana, C.; Padmanabhan, S.; Kabalka, G. W. Tetrahedron Lett. 1990, 31, 6977. (e) Trost, B. M.; Lee, C. B.; Weiss, J. M. J. Am. Chem. Soc. 1995, 117, 7247. (f) Sydnes, L. K.; Sandberg, M. Tetrahedron 1997, 53, 12679. (g) Sandberg, M.; Sydnes, L. K. Tetrahedron Lett. 1998, 39, 6361. (h) Sandberg, M.; Sydnes, L. K. Org. Lett. 2000, 2, 687. (i) Trost, B. M.; Lee, C. B. J. Am. Chem. Soc. 2001, 123, 12191.

5. Frick, J. G.; Harper, R. J. J. Appl. Polym. Sci. 1984, 29, 1433.

6. Eanderson, W. R. Eur. Pat. Appl. EP.125, 781, 1985; Chem. Abstr. 1985, 102, P64010K.

7. (a) Fry, A. J.; Rho, A. K.; Sherman, L. R.; Sherwin, C. S. J. Org. Chem. 1991, 56, 3283. (b) Bhatia, B.; Punniyamurthy, T.; Iqbal, J. J. Org. Chem. 1993, 58, 5518. (c) Bandgar, B. P. N.; Mahajan, P.; Mulay, D. P.; Thote, J. L.; Wadgaonkar, P. P. J. Chem. Res. (S), 1995, 67, 470. (d) Raju, S. V. N. J. Chem. Res. (S), 1996, 68, 470. (e) Deka, N.; Kalita, D. J.; Borah, R.; Sarma, J. C. J. Org. Chem. 1997, 62, 1563. (f) Aggarwal, V. K.; Fonquerna, S.; Vennall, G. P. Synlett 1998, 849. (g) Ballini, R.; Bordoni, M.; Bosica, G.; Maggi, R.; Sartori, G. 
Tetrahedron Lett. 1998, 39, 7587. (h) Chandra, K. L.; Saravanan, P.; Singh, V. K. Synlett 2000, 359. (i) Karimi, B.; Seradj, H.; Ebrahimian, G. R. Synlett 2000, 623. (j) Carrigan, M. D.; Eash, K. J.; Oswald, M. C.; Mohan, R. S. Tetrahedron Lett. 2001, 42, 8133. (k) Sumida, N.; Nishioka, K.; Sato, T. Synlett 2001, 1921. (1) Trost, B. M.; Lee, C. B. J. Am. Chem. Soc. 2001, 123, 3671. (m) Yadav, J. S.; Reddy, B. V. S.; Srinivas, C. Synth. Commun. 2002, 32, 1175. (n) Yadav, J. S.; Reddy, B. V. S.; Venugopal, C.; Ramalingam, T. Synlett 2002, 604. (o) Curini, M.; Epifano, F.; Marcotullio, M. C.; Rosati, O.; Nocchetti, M. Tetrahedron Lett. 2002, 43, 2709. (p) Roy, S. C.; Banerjee, B. Synlett 2002, 1677. (q) Romanelli, G. P.; Thomas, H. J.; Baronetti, G. T.; Autino J. C. Tetrahedron Lett. 2003, 44, 1301. (r) Firouzabadi, H.; Iranpoor, N.; Nowrouzi, F.; Amani, K. Tetrahedron Lett. 2003, 44, 3951. (s) Smitha, G.; Reddy, C. S. Tetrahedron 2003, 59, 9571. (t) Karimi, B.; Maleki J. J. Org. Chem. 2003, 68, 4951. (u) Aggen, D. H. Arnold, J. N.; Hayes, P. D.; Smoter, N. J.; Mohan, R. S. Tetrahedron 2004, 60, 3675. (v) Chakraborti, A. S.; Thilagavathi, R.; Kumar, R. Synthesis 2004, 831. (w) Yin, L.; Zhang, Z. H.; Wang, Y. M.; Pang M. L. Synlett 2004, 1727. (x) Ishino, Y.; Mihara, M.; Takeuchi, T.; Takemoto, M. Tetrahedron Lett. 2004, 45, 3503. (y) Ghosh, R.; Maiti, S.; Chakraborty, A.; Halder, R. J. Mol. Catal. A: Chem. 2004, 215, 49. (z) Zhang, Z. H.; Yin, L.; Li, Y.; Wang, Y. M. Tetrahedron Lett. 2005, 46, 889.

8. Hajipour, A. R.; Zarei, A.; Khazdooz, L.; Mirjalili, B. B. F.; Sheikhan, N.; Zahmatkesh, S.; Ruohoa, A. E. Synthesis 2005, 3644.

9. (a) Bartoli, G.; Cupone, G.; Dalpozzo, R.; De Nino, A.; Maiuolo, L.; Procopio, A.; Sambri, L.; Tagarelli, A. Tetrahedron Lett. 2002, 43, 5945. (b) Dalpozzo, R.; De Nino, A.; Maiuolo, L.; Procopio, A.; Tagarelli, A.; Sindona, G.; Bartoli, G. J. Org. Chem. 2002, 67, 9093. (c) Bartoli, G.; Bosco, M.; Dalpozzo, R.; Giuliani, A.; Marcantoni, E.; Mecozzi, T.; Sambri, L.; Torregiani, E. J. Org. Chem. 2002, 67, 9111. (d) Dalpozzo, R.; De Nino, A.; Maiuolo, L.; Procopio, A.; Nardi, M.; Bartoli, G.; Romeo R. Tetrahedron Lett. 2003, 44, 5621 (e) Bartoli, G.; Marcantoni, E.; Sambri, L. Synlett 2003, 2101. (f) Bartoli, G.; Bosco, M.; Dalpozzo, R.; Marcantoni, E.; Massaccesi, M.; Sambri, L. Eur. J. Org. Chem. 2003, 4611. (g) Bartoli, G.; Dalpozzo, R.; De Nino, A.; Maiuolo, L.; Nardi, M.; Procopio, A.; Tagarelli A. Green Chem. 2004, 6, 191. (h) Bartoli, G.; Dalpozzo, R.; De Nino, A.; Maiuolo, L.; Nardi, M.; Procopio, A.; Tagarelli A. Eur. J. Org. Chem. 2004, 2176. (i) Bartoli, G.; De Nino, A.; Dalpozzo, R.; Maiuolo, L.; Nardi, M.; Procopio, A.; Tagarelli A. Lett. Org. Chem. 2005, 2, 51.

10. Kobayashi, S.; Sugiura, M.; Kitagawa, H.; Lam, W. W. L. Chem. Rev. 2002, 102, 2227.

11. Tsuruta H.; Yamaguchi K.; Imamoto T. Tetrahedron 2003, 59, 10419.

12. Kobayashi, S.; Manabe, K. Acc. Chem. Res. 2002, 35, 209.

13. Procopio, A.; Dalpozzo, R.; De Nino, A.; Maiuolo, L.; Russo, B.; Sindona, G. Adv. Synth. Catal. 2004, 346, 1465.

14. (a) Dalpozzo, R.; De Nino, A.; Maiuolo, L.; Nardi, M.; Procopio, A.; Tagarelli, A. Synthesis 2004, 496. (b) Procopio, A.; Dalpozzo, R.; De Nino, A.; Maiuolo, L.; Nardi M.; Romeo, G. Org. Biomol. Chem. 2005, 4129. 
15. Procopio, A.; Dalpozzo, R.; De Nino, A.; Nardi, M.; Sindona, G.; Tagarelli, A. Synlett 2004, 2633. (b) Procopio, A.; Dalpozzo, R.; De Nino, A.; Maiuolo, L.; Nardi, M.; Russo, B. Adv. Synth. Catal. 2005, 347, 1447.

16. Dalpozzo, R.; De Nino, A.; Maiuolo, L.; Nardi, M.; Russo, B.; Procopio, A. Synthesis 2006, 332.

17. We have not investigated the real reason of this deactivation, and the cited reactions were not helpful. We think that the nitrogen atom can scavenge metal ion, preventing its catalytic activity.

18. Hirao, T.; Santhitikul, S.; Takeuchi, H.; Ogawa, A.; Sakurai, H. Tetrahedron 2003, 59, 10147.

19. We think that the enol ester arises from heating into the injector port of the GC-MS, since workup of the reaction, after heating, did not hydrolyse the enol ester.

20. We think that the required longer reaction times and the low stability of the product are the reasons why other authors did not find any reaction with ketones.

21. Norman, R. O. C.; Thomas C. B. J. Chem. Soc. B 1967, 771.

22. Fujita, M.; Kim, W. H.; Sakanishi, Y.; Fujiwara, K.; Hirayama, S.; Okuyama, T.; Ohki, Y.; Tatsumi, K.; Yoshioka, Y. J. Am. Chem. Soc. 2004, 126, 7548.

23. Noji, M.; Ohno, T.; Fuji, K.; Futaba, N.; Tajima, H.; Ishii, K. J. Org. Chem. 2003, 68, 9340. 